\title{
Pancreatic pseudocyst with complicating colonic fistula successfully closed using the over-the-scope-clip system
}
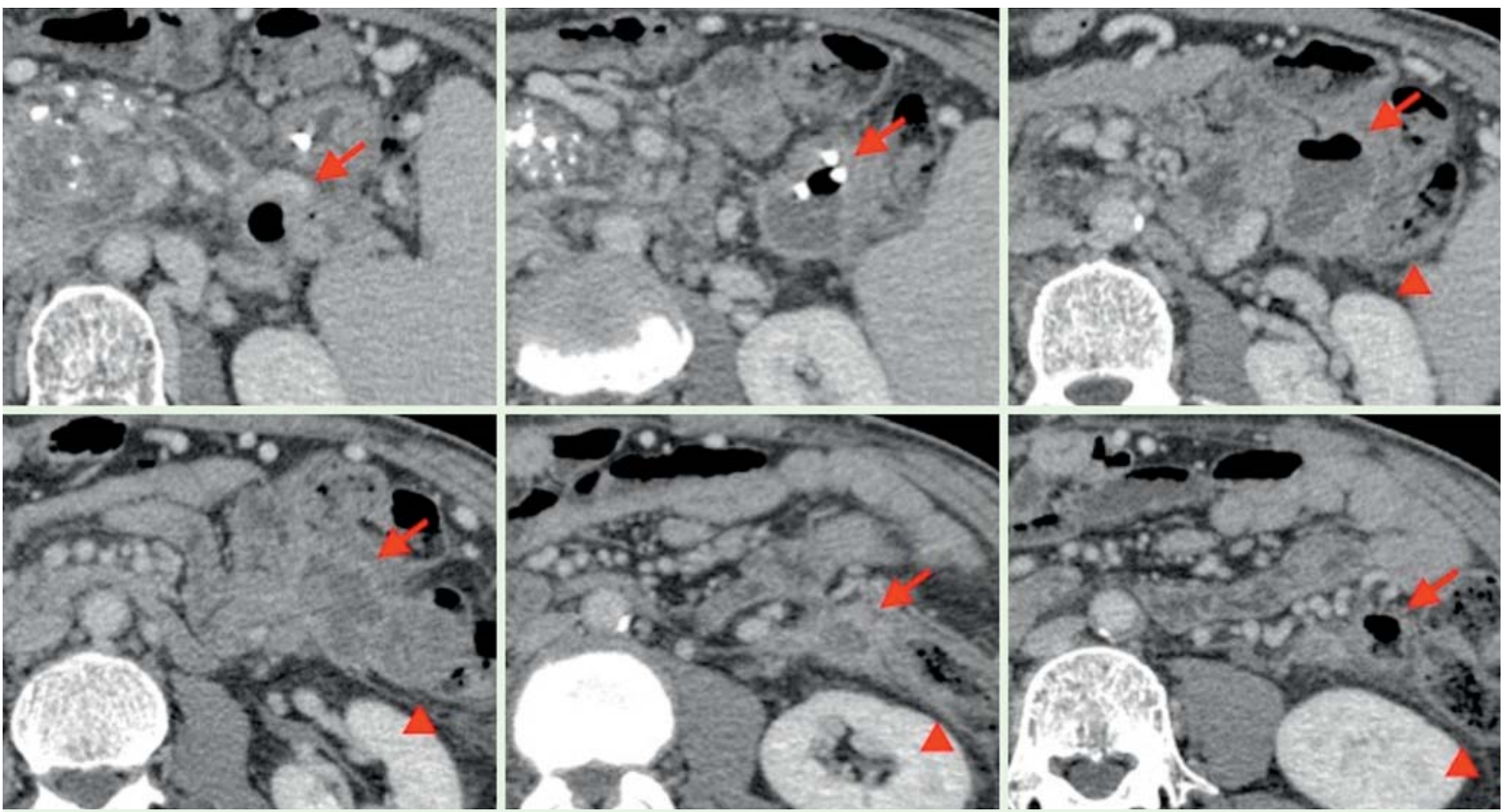

Fig. 1 Abdominal computed tomography (CT) examination revealed obstruction of the main pancreatic duct in the pancreatic tail, and formation of a pseudocyst caudal to this obstruction. The wall of the pseudocyst was thickened, and air bubbles were observed inside the cyst, indicating infection of the cyst (arrow). A section of the border with the descending colon (arrowhead) was obscured, and therefore, the presence of a fistula was suspected.

A 53-year-old man had a history of alcohol-induced chronic pancreatitis. In July 2011 , because of suspicion of a pseudocyst infection, he had undergone endoscopic ultrasound (EUS)-guided cyst drainage. Further investigation after drainage had revealed a fistula between the pancreatic pseudocyst and descending colon; however, the transgastric internal drainage tube was left in place because of the severity of the patient's condition. In August 2013, the patient visited our institution again and on the basis of an abdominal computed tomography (CT) examination, it was suspected that the fistula between the pancreatic pseudocyst and colon had persisted and was re-infected $(\bullet$ Fig. 1). The transgastric internal drainage tube that had been inserted during his previous hospitalization was found to be obstructed ( Fig. 2). Although the general health of the patient had improved, the fistula between the pancreatic pseudocyst and colon was still present ( $\bullet$ Fig. 3). This fistula was believed to have caused repeated cyst infections. Therefore, using lower

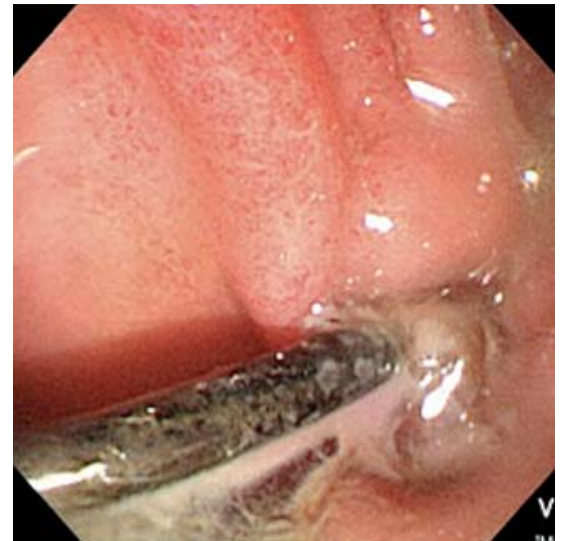

Fig. 2 The internal drainage tube that had been transgastrically inserted into the pancreatic pseudocyst during the patient's previous hospitalization was obstructed, with evidence of pus outflow from the insertion site.

gastrointestinal endoscopy, the location of the fistula in the descending colon was confirmed ( Fig.4) and the fistula was closed using the over-the-scope clip (OTSC) system (Ovesco Endoscopy AG, Tübingen, Germany) ( Fig.5). Thereafter,

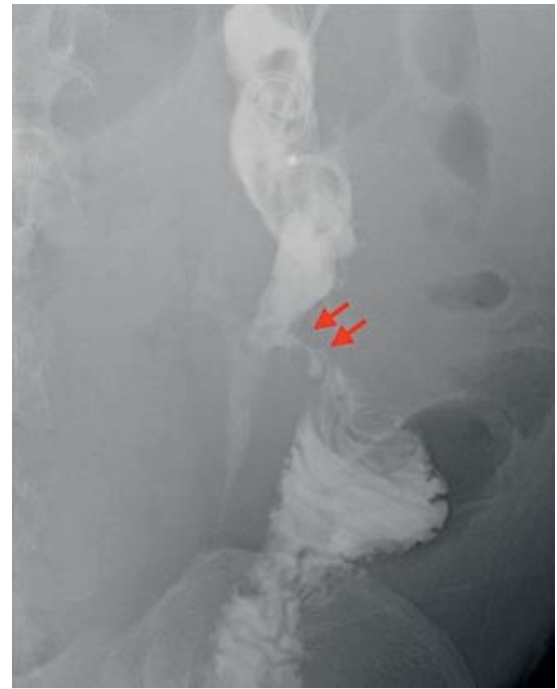

Fig. 3 Transgastric insertion of a nasal drainage catheter into the pseudocyst allowed visualization of a fistula between the pancreatic pseudocyst and descending colon (arrow).

fistula closure was confirmed by radiographic imaging of the transnasal catheter that had been inserted ( Fig.6). At the 


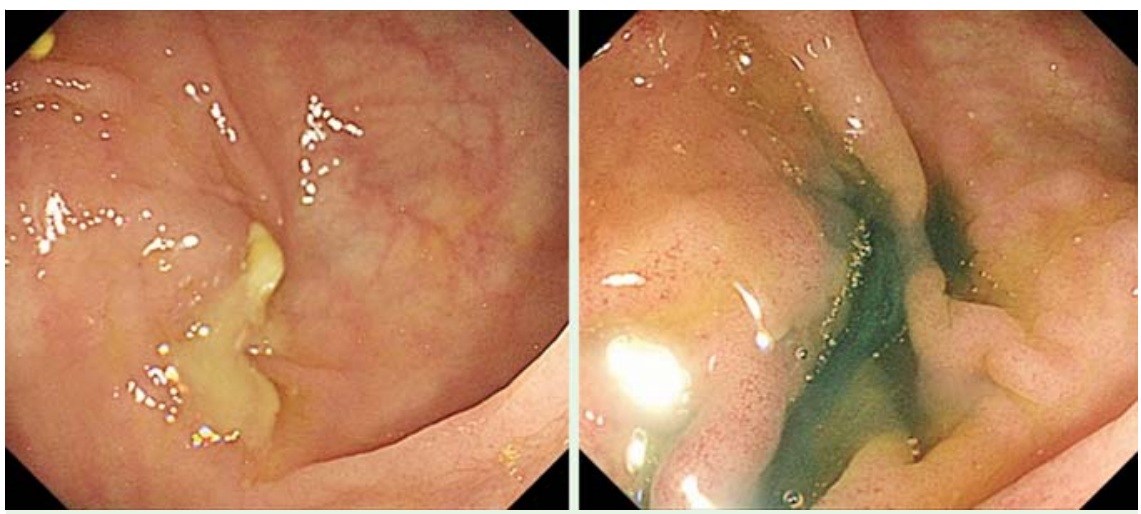

Fig. 4 The location of the opening of the fistula in the descending colon was confirmed using lower gastrointestinal endoscopy. Pus outflow from the opening of the fistula was observed (left panel). Outflow from the fistula was observed when indigo carmine was injected through the transnasal drainage catheter (right panel).
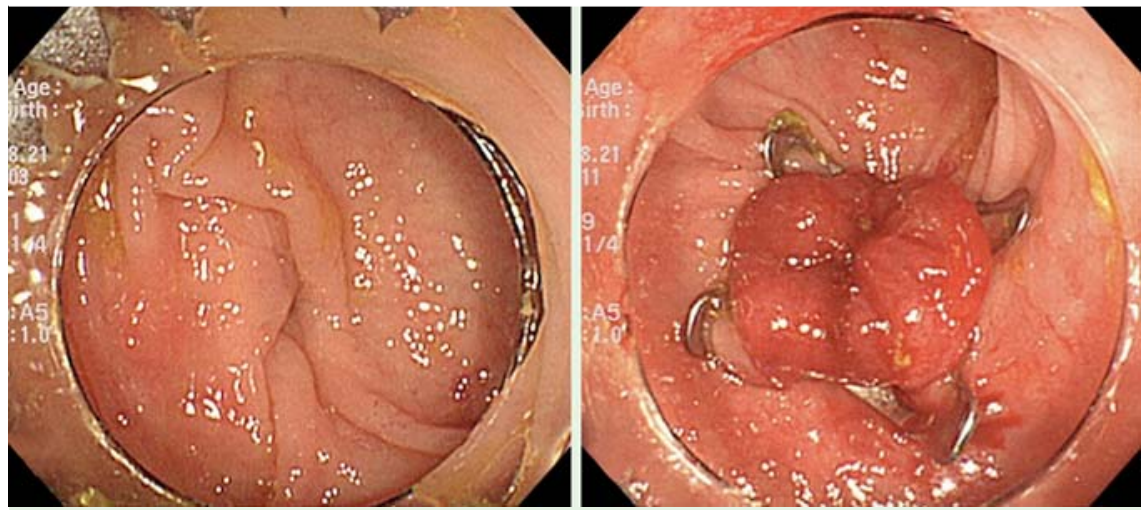

Fig. 5 The fistula between the pancreatic pseudocyst and descending colon was closed using the over-the-scope clip (OTSC) system.

time of writing, the patient has two internal drainage tubes in place and is under observation, but the pancreatic pseudocyst has decreased in size, and no signs of reinfection have been reported.

Although there is no standard consensus regarding the treatment of pancreatic pseudocysts with a colonic fistula, some recent studies have reported successful closure of a fistula using only endoscopic drainage in order to avoid surgery $[1,2]$. In the present case, the cyst showed repeated infections after endoscopic drainage and it was determined that fistula closure would be necessary but would be difficult with endoscopic drainage alone; thus, the OTSC system was used. Although very few case reports have documented the use of the OTSC system to close a colonic fistula associated with acute pancreatitis $[3,4]$, the system is less invasive than surgery and can be considered as a treatment option.

Endoscopy_UCTN_Code_TTT_1AR_2AG

\section{Competing interests: None}

\section{Yuta Koike, Takahiko Kudo, Taku Shigesawa, Yomo Fujita, Ayana Endo, Yuji Ono, Michio Nakamura, Atsushi Nagasaka, Shuji Nishikawa}

Department of Gastroenterology, Sapporo City General Hospital, Sapporo, Japan

\section{References}

1 Howell DA, Dy RM, Gerstein WH et al. Infected pancreatic pseudocyst with colonic fistula formation successfully managed by endoscopic drainage alone: report of two cases. AM J Gastoenterol 2000; 95: 1821 - 1823

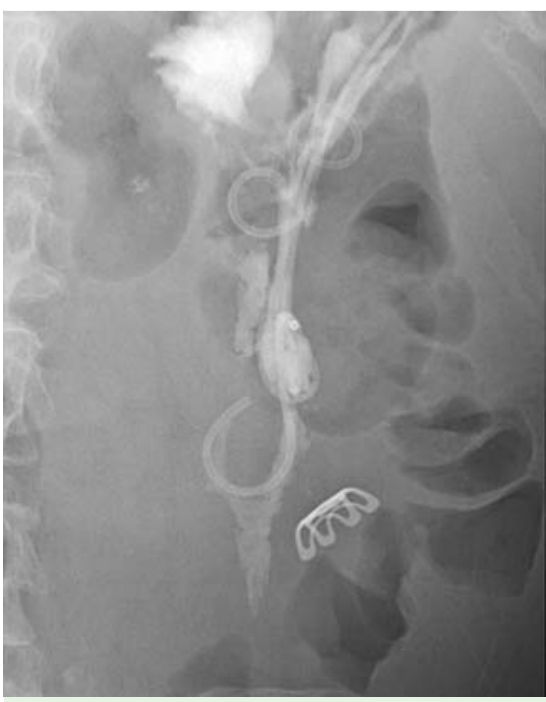

Fig. 6 After use of the over-the-scope clip (OTSC) system, complete closure of the fistula was confirmed by radiographic imaging of the transnasal catheter. The patient has two internal drainage tubes in place and is under observation, but the pancreatic pseudocyst has decreased in size, and no signs of reinfection have been reported.

2 Fujii K, Suzuki K, Goto Y et al. Pancreatic-colonic fistula successfully treated with endoscopic transpapillary nasopancreatic drainage. Endoscopy 2011; 43: E154 -E155

3 Gray DM 2nd, Mullady DK. Attempted endoscopic closure of a pancreaticocolonic fistula with an over-the-scope-clip. JOP 2012; 13 : $712-714$

4 Ito $K$, Igarashi $Y$, Okano $N$ et al. Severe acute pancreatitis with complicating colonic fistula successfully closed using the over-thescope clip system. Case Rep Gastroenterol 2013; 7: 314-321

Bibliography

DOI http://dx.doi.org/

10.1055/s-0034-1365106

Endoscopy 2014; 46: E178-E179

(c) Georg Thieme Verlag KG

Stuttgart · New York

ISSN 0013-726X

\section{Corresponding author}

\section{Y. Koike, MD}

Department of Gastroenterology, Sapporo City General Hospital

Kita 13 Nishi 11 1-1, Tyuou-ku

Sapporo, 060-0011

Japan

Fax: +81-011-726-7912

yutakoike1217@gmail.com 